\title{
Rectal polyp as presentation form of colitis cystica profunda
}

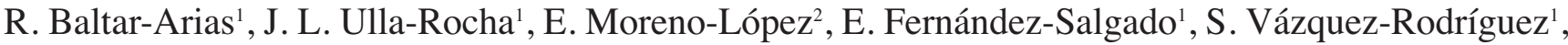 \\ W. Díaz-Saa ${ }^{1}$, V. Carrera-González ${ }^{1}$ and E. Vázquez-Astray ${ }^{1}$
}

Departments of ${ }^{l}$ Gastroenterology, and ${ }^{2}$ Anesthesiology. Complexo Hospitalario de Pontevedra. Spain

\section{CASE REPORT}

A 63-year-old woman complained of rectal bleeding of hemorroidal type and spontaneous emission of mucus in the feces for 2 months. Laboratory tests, tumoral markers included, were normal. A colonoscopy was performed revealing a 2-cm polypoid lesion (Fig. 1A) with a mucoid and gelatinous material (Fig. 2) coming out of it. Cytology and biopsies were inconclusive. An endoscopic ultrasonogram showed a cystic anechoic 20 -mm lesion filled with some non-solid ma-

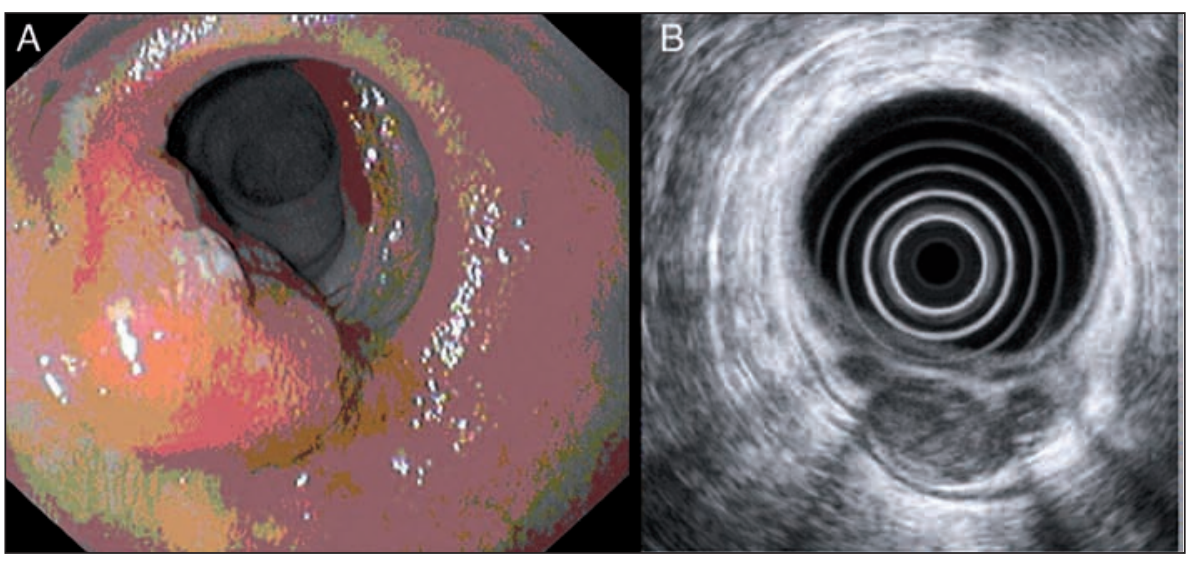

Fig. 1. 2-cm rectal submucosal lesion (A). On transrectal ultrasonography, cystic lesion of $15 \times 25$ $\mathrm{mm}$ located in the submucosal layer (B).

Lesión rectal de $2 \mathrm{~cm}$ y aspecto submucoso (A). En ecoendoscopia, lesión quística de 15 × 25 mm localizada en la capa submucosa (B).

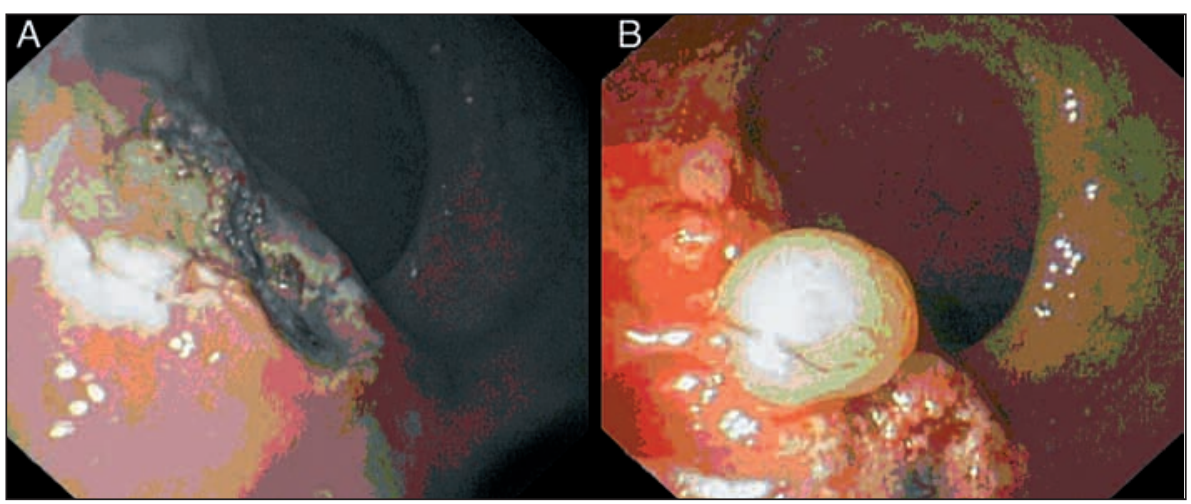

Fig. 2. Mucosal resection of the upper portion of the lesion (A). Mucoid material coming out after taking biopsies (B).

Resección mucosa de la porción superior de la lesión (A). Salida de material mucoide tras la toma de biopsias (B). terials located in the submucosal layer of the rectal wall (Fig. 1B), without adjacent adenopathies, and a hypertonic internal anal sphincter. Histology showed mucosal thickening with dilated glands and the presence of inflammatory infiltrates, muscular fibers, and congestive vessels in the muscularis propria, findings that can be seen in rectal prolapse. These results, in addition to endoscopic and ultrasonographic findings, established the diagnosis.

\section{DISCUSSION}

Colitis cystica profunda is a rare and benign condition characterized by mucoid cysts in the submucosal layer of the colon (1), mainly in the anterior rectal wall. It is associated with rectal prolapse, isolated rectal ulcer, inflammatory bowel disease, and local trauma (2). Typical symptoms are hematochezia, mucus in the feces, and diarrhea (3). The diagnosis is based on endoscopy, computer tomography, and magnetic resonance imaging. Colonoscopy shows polypoid or sessile lesions covered with normal, edematous or congestive mucosa, or ulcerated areas (4). On transrectal ultrasonography multiple submucosal cysts with hyperechoic bands corresponding to fibros- 
al areas among them can be seen, without nodal or muscular layer involvement (5). Treatment is conservative (3), except for highly symptomatic cases where surgery is mandatory.

\title{
REFERENCES
}

1. Kornprat P, Langner C, Pfeifer J, Mischinger HJ. Colitis cystica profunda associated with rectal prolapse: report of a case. Int J Colorectal Dis 2007; 22 (12): $1555-6$.

2. De Toro G, Villaseca M, Roa JC. Colitis quística profunda posradioterapia. Caso clínico. Rev Med Chile 2007; 135: 759-63.

3. Higuera Álvarez R, De la Peña García J, San Miguel G, Castro B. Colitis cystica profunda. Rev Esp Enferm Dig 2008; 100: $240-2$.

4. Hulsmans FJH, Lok Tio T, Reeders JWAJ, Tytgat GNJ. Transrectal US in the diagnosis of localized colitis cystica profunda. Radiology 1991; 181: 201-3.

5. Valenzuela M, Martín-Ruiz JL, Alvarez-Cienfuegos E, Caballero AM, Gallego F, Carmona I, et al. Colitis cystica profunda: imaging diagnosis and conservative treatment. Report of two cases. Dis Colon Rectum 1996; 39: 587-90.

\section{Pólipo rectal como forma de presentación de colitis quística profunda}

\author{
R. Baltar Arias¹, J. L. Ulla Rochaㄹ, E. Moreno López², E. Fernández Salgado¹, S. Vázquez Rodríguez ${ }^{1}$, \\ W. Díaz Saa ${ }^{1}$, V. Carrera González y E. Vázquez Astray ${ }^{1}$
}

Servicios de ${ }^{1}$ Aparato Digestivo y ${ }^{2}$ Anestesia y Reanimación. Complexo Hospitalario de Pontevedra

\section{CASO CLÍNICO}

Mujer de 63 años presenta rectorragias de perfil hemorroidal y eliminación de mucosidad clara con las heces de aproximadamente 2 meses de evolución. La analítica de sangre con marcadores tumorales es normal. Se realiza una colonoscopia visualizando una lesión vegetante de $2 \mathrm{~cm}$ y aspecto polipoide (Fig. 1A) de la que sale un material mucoide y gelatinoso (Fig. 2). La citología y las biopsias resultan negativas. Posteriormente se practica una ecoendoscopia alta donde se evidencia una formación quística anecoica de $20 \mathrm{~mm}$ con algún grumo en su interior, localizada en la capa submucosa de la pared rectal (Fig. 1B), sin adenopatías adyacentes, además de un esfínter anal interno hipertrófico. La histología revela un engrosamiento mucoso con dilatación glandular y la presencia de un infiltrado inflamatorio, fibras musculares y vasos congestivos en la lámina propia, datos compatibles con prolapso mucoso rectal. Estos hallazgos, junto con los endoscópicos y ecoendoscópicos, determinan el diagnóstico.

\section{DISCUSIÓN}

Es una entidad infrecuente y benigna caracterizada por la presencia de quistes mucoides en la capa submucosa del colon (1), preferentemente en la pared rectal anterior. Se asocia con el prolapso mucoso, la úlcera rectal solitaria, la enfermedad inflamatoria intestinal y los traumatismos (2). Los síntomas típicos de presentación son la hematoquecia, la presencia de moco en las heces y la diarrea (3). El diagnóstico se alcanza mediante pruebas endoscópicas, TAC y RMN. La colonoscopia muestra lesiones polipoides o sesiles cubiertas de mucosa normal, edematosa o congestiva, o áreas ulceradas (4). En la ecoendoscopia se suelen visualizar múltiples quistes submucosos entre los que se observan bandas hiperecogénicas correspondientes a zonas de fibrosis, sin afectación de ganglios ni de la capa muscular (5). El tratamiento es conservador (3), salvo en casos muy sintomáticos en los que es necesaria la cirugía. 